\title{
Value of soluble Urokinase plasminogen activator receptor over age as a biomarker of impaired myocardial relaxation
}

\author{
Angela S. Koh ${ }^{1,2^{*}}$, Bhaarathy Velmurugan ${ }^{3}$, Fei Gao ${ }^{1,2}$, Ru San Tan ${ }^{1,2}$, Jia-Ing Wong ${ }^{1}$, Louis L. Y. Teo ${ }^{1}$,
} Bryan M. H. Keng ${ }^{1}$, Serene J. M. Chua ${ }^{1}$, Jian-Min Yuan ${ }^{4,5}$, Woon-Puay Koh ${ }^{2,6}$ and Christine Cheung ${ }^{3,7}$

\begin{abstract}
Background: SUPAR is a biomarker that reflects the level of immune activation. As inflammation plays an important role in the ageing process of the cardiovascular system, we hypothesized that suPAR might be a useful predictive biomarker of the ageing heart.

Methods: We performed conventional and tissue Doppler echocardiography and measured plasma suPAR levels.

Results: We studied community adults ( $n=120,37.5 \%$ female) (mean age: $70.3 \pm 9.3$ years) without known cardiovascular disease (CVD). Participants with impaired myocardial relaxation were older ( $84 \%$ vs $59 \%$ were aged $\geq 71$ years, $p=0.002$ ), with more diabetes mellitus ( $27 \%$ vs $11 \%, p=0.034)$. SuPAR levels were higher among participants with impaired myocardial relaxation ( $3.9 \mathrm{ng} / \mathrm{ml}$ vs $3.0 \mathrm{ng} / \mathrm{ml}, p=0.015)$. At the univariate level, older age (OR 3.6; $95 \% \mathrm{Cl} 1.6,8.5 ; p=0.003$ ), diabetes mellitus (OR 3.04; 95\%Cl 1.1, 8.8; $p=0.04)$, systolic blood pressure (OR 1.03; 95\%Cl 1.001, 1.1; $p=0.041)$ and suPAR levels $\geq 3.00 \mathrm{ng} / \mathrm{ml}$ (OR 3.4; 95\%Cl 1.16, 7.4; $p=0.002)$ were associated with impaired myocardial relaxation. In multivariable regression analysis, only older age (OR 2.8; 95\%Cl 1.1, 6.7; $p=0.026$ ) and suPAR (OR 2.7; 95\%Cl 1.2, 6.1; $p=0.018)$ remained independently associated with impaired myocardial relaxation. Receiver operating characteristics (ROC) curve analysis revealed an area under the curve (AUC) value of $0.63(95 \% \mathrm{Cl} 0.54,0.71)$ for model that included age alone. Addition of suPAR significantly increased AUC value to $0.70(95 \% \mathrm{Cl} 0.60,0.79)$, which was significantly larger than the model with age alone $(p=0.016)$.
\end{abstract}

Conclusion: We demonstrate additional ability of suPAR, over age, to predict impaired myocardial relaxation.

Trial registration: ClinicalTrials.gov Identifier: NCT02791139 (Registered May 31, 2016).

Keywords: Soluble urokinase plasminogen activator receptor, Biomarker, Cardiac function, Cardiovascular disease, Elderly, Ageing

\section{Background}

Age-associated changes in cardiovascular (CV) structure and function and the clinical implications of cardiovascular ageing are well established [1-3]. In spite of this knowledge, clinicians have limited tools to 'detect' CV ageing, and hence the ability to focus therapeutic efforts on CV ageing is hampered. Many of the pathophysiological changes of age-associated $\mathrm{CV}$ changes cannot be

\footnotetext{
* Correspondence: angela.koh.s.m@nhcs.com.sg

${ }^{1}$ National Heart Centre Singapore, 5 Hospital Drive, Singapore 169609, Singapore

${ }^{2}$ Duke-NUS Medical School, Singapore, Singapore

Full list of author information is available at the end of the article
}

detected in the blood. This is in contrast to traditional $\mathrm{CV}$ risk factors such as diabetes mellitus and dyslipidemia that have blood-based diagnostic tools.

A chronic inflammatory state mediated by mechanical and humoral factors, is commonly described in ageingrelated cardiovascular disease. These proinflammatory mechanisms drive age-associated arterial remodelling and incomplete myocardial relaxation at the onset of diastole, reducing early left ventricular filling rate $[4,5]$. A potential blood-based biomarker reflective of this state of chronic inflammation and which is relatively stable, is soluble urokinase plasminogen activator receptor (suPAR) [6]. SuPAR is emerging as a useful biomarker reflective of increased 
inflammation particularly in cardiovascular cohorts [7-9]. SuPAR originates from the proteolytic cleavage of the membrane-bound receptor (urokinase plasminogen activator receptor, uPAR) from activated immune and endothelial cells and reflects the level of immune activation [10]. As inflammation plays an important role in the pathogenesis of age-related CVD, we hypothesize that suPAR might be of value as a blood-based biomarker, prior to CVD development. This will have immense value in future trials to target 'age' as a risk factor, using suPAR as a blood-based target for such interventions.

In this study, we investigated the value of suPAR as an inflammatory biomarker for ageing-related impairments in myocardial relaxation, among elderly community participants without clinical CVD.

\section{Methods}

The subjects were recruited from the Cardiac Aging Study (CAS), a prospective study initiated in 2014 that examines characteristics and determinants of cardiovascular function in elderly adults. CAS participants were recruited from the prospective, population-based cohort, the Singapore Chinese Health Study (SCHS) [11] and directly from the local community. Briefly, the SCHS recruited 63,257 adults aged 45-74 years and residing in Singapore between 1993 and 1998 for long-term study on association between lifestyle factors and disease risks. This study recruited surviving cohort participants who were re-contacted between 2014 and 2016 for Follow-up III, who did not have self-reported history of physiciandiagnosed cardiovascular disease (such as coronary heart disease or stroke).

The study sample consisted of men and women who participated in the baseline CAS 2014 examination who had no self-reported history of physician-diagnosed cardiovascular disease (such as coronary heart disease, atrial fibrillation), stroke or cancer. Written informed consent was obtained from participants upon enrolment. The SingHealth Centralised Institutional Review Board had approved the study protocol.

All participants were examined and interviewed on one study visit by trained study coordinators. Participants completed a standardized questionnaire that included medical history and coronary risk factors. Hypertension was defined by current use of antihypertensive drugs or physiciandiagnosed hypertension. Diabetes mellitus was defined by current use of anti-diabetic agents or physician-diagnosed diabetes mellitus. Dyslipidemia was defined by current use of lipid-lowering agents or physician-diagnosed dyslipidemia. Smoking history was defined as ever smokers (former or current smoking) or never smokers. Body mass index was calculated as weight in kilograms divided by the square of height in meters. Sinus rhythm status was ascertained by resting electrocardiogram. Clinical data were obtained on the same day as assessment of echocardiography and serum collection.

Using a ALOKA $\alpha 10$ with a $3.5 \mathrm{MHz}$ probe, echocardiography was performed. Standard echocardiography including 2-D, M-mode, pulse Doppler and tissue Doppler imaging, was performed in the standard parasternal and apical (apical 4-chamber, apical 2-chamber and apical long) views, over three cardiac cycles for each subject. Left ventricular ejection fraction (LVEF), left atrial (LA) volume and LA volume index were obtained. Using Doppler echocardiography, trans-mitral flow E and A wave with the sample volume position at the tip of the mitral valve leaflets from the apical 4-chamber view was recorded. Using sample volume at the septal and lateral annulus from apical four chamber view, we obtained pulsed wave tissue Doppler signals. The frame rate was between 80 and 100 frames per second. Systolic, early diastolic and atrial velocities were recorded and expressed as $\mathrm{S}^{\prime}, \mathrm{E}$,' and A, respectively'. All measurements were measured by the same operator (i.e., JIW) and the measurements were averaged over three cardiac cycles.

Antecubital venous blood samples $(20-30 \mathrm{ml})$ were taken from consenting participants in the morning; fasting was not required before blood collection. After collection, the blood samples were immediately placed on ice for transportation and were processed within $6 \mathrm{~h}$ to obtain serum samples, which were subsequently stored at $-80^{\circ} \mathrm{C}$.

Plasma levels of soluble UPAR were measured using the Human uPAR Quantikine ELISA kit (R\&D Systems, cat no. DUP00), according to the manufacturer's assay procedures. Briefly, $100 \mu \mathrm{L}$ of assay diluent was added to each well, followed by $50 \mu \mathrm{L}$ of plasma samples or protein standards. The plate was sealed and incubated at room temperature for $2 \mathrm{~h}$. Subsequently, the plate was washed 4 times before $200 \mu \mathrm{L}$ of conjugate was added to each well, then incubated for another $2 \mathrm{~h}$ at room temperature. Thereafter, the plate was washed again 4 times. $200 \mu \mathrm{L}$ of substrate solution was then added and incubated for $30 \mathrm{~min}$, away from light. $50 \mu \mathrm{L}$ of stop solution was added and read at $450 \mathrm{~nm}$ within $30 \mathrm{~min}$ in order to halt substrate development. We calculated concentration of suPAR from the equation generated from the standard curve.

In this cohort, the mean $\mathrm{E}$ to $\mathrm{A}$ ratio was 0.84 . We used $\mathrm{E}$ to A ratio 0.84 as a cut off to define impaired myocardial relaxation and grouped suPAR using its mean value in the group without impaired myocardial relaxation.

We performed bivariate comparisons between subjects with and without impaired myocardial relaxation with the $\mathrm{t}$ test for continuous variables and the chi-square test for categorical variables. Continuous variables are reported as a mean with standard deviation (SD).

We constructed multivariable logistic regression models to assess the association of suPAR with impaired 
myocardial relaxation. The initial univariate logistic regression model examined the individual association with demographic variables and existing medical conditions. Those associated in the univariate analysis with a $p<0.05$ were candidate suPAR effect modifiers. Then the potential effect medications of these candidates were assessed individually using multivariable logistic regression.

We further evaluated the discrimination of the models by calculating the area under the receiver-operating characteristic (ROC).

All analyses were performed in STATA 13.

\section{Results}

A total of 120 participants (mean age $70.3 \pm 9.3$ years; 45 women) were included in this analysis. All completed transthoracic echocardiography and blood sampling on the same day. Baseline clinical characteristics of the study sample are presented in Table 1 .

Participants with impaired myocardial relaxation were older $(83.8 \%$ vs $58.7 \%$ were aged $\geq 71$ years, $p=0.002)$, with more diabetes mellitus $(27.0 \%$ vs $11.0 \%, p=0.034)$. Participants with impaired myocardial relaxation had larger septal wall thickness at end diastole (IVSD) $(0.9 \pm 0.2$ vs $0.8 \pm 0.1), p=0.012$ ), smaller left ventricular internal diameter at end systole (LVIDS) $(2.5 \pm 0.4$ vs $2.6 \pm 0.4$, $p=0.027$ ), higher left ventricular ejection fraction (LVEF) (75.3\% vs $72.7 \%, p=0.047$ ), lower peak early phase filling velocity $(0.6 \pm 0.1$ vs $0.7 \pm 0.2, p=0.0001)$, higher peak atrial phase filling velocity $(0.8 \pm 0.2$ vs $0.7 \pm 0.2, p=0.0014)$, lower E/A ratio $(0.8 \pm 0.2$ vs $1.1 \pm 0.4, p<0.0001)$, higher E wave deceleration time $(218.0 \pm 39.0$ vs $198.0 \pm 37.1$, $p=0.0064)$ and lower peak early diastolic septal mitral annular velocity $(0.06 \pm 0.02$ vs $0.09 \pm 0.02, p<0.0001)$. SuPAR levels were higher among participants with impaired myocardial relaxation $(3.9 \pm 2.2 \mathrm{ng} / \mathrm{ml}$ vs $3.0 \pm$ $1.4 \mathrm{ng} / \mathrm{ml}, p=0.015$ ).

At the univariate level, older age (OR 3.6, 95\%CI 1.6, 8.5; $p=0.003$ ), diabetes mellitus (OR 3.04, 95\%CI 1.1, $8.8 ; p=0.040)$, systolic blood pressure (OR $1.03,95 \% \mathrm{CI}$ $1.001,1.1 ; p=0.041$ ) and suPAR levels $\geq 3.00 \mathrm{ng} / \mathrm{ml}$ (OR 3.4; 95\%CI 1.6, 7.4; $p=0.002$ ) were associated with impaired myocardial relaxation. After adjusting for age, diabetes mellitus and systolic blood pressure respectively, suPAR remained independently associated with impaired myocardial relaxation (all OR $>2.5 ; p<0.05$ ) (Table 2).

Figure 1 shows that combining suPAR and age increased $(p=0.016)$ the area under the curve (AUC) increased from $0.63(95 \%$ CI $0.54,0.71)$ to 0.70 (95\% CI $0.60,0.79)$.

\section{Discussion}

In this study, we analysed the value of suPAR levels in predicting ageing-related impairments in myocardial relaxation. We provide novel evidence that suPAR adds incremental value over age, in predicting impaired myocardial relaxation that is commonly observed in aged cohorts. This suggests that suPAR is a potential candidate that can be used as a blood-based biomarker of the ageing heart, superior to age.

Our study participants were predominantly old without clinical cardiovascular disease. The impairments in myocardial relaxation were associated with chronological ageing, independent of risk factors. Therefore, our data fills an important gap in geriatric cardiology, where in the lack of blood-based tools to predict risk of ageingrelated myocardial dysfunction is hampering clinicians' ability to identify elderly with early alterations in myocardial function.

In the area of early diastolic dysfunction, hardly any study has looked at the association between suPAR and subclinical myocardial dysfunction. One recent study investigated associations between suPAR and subclinical diastolic dysfunction among a cohort wholly composed of Type 1 diabetic patients [12]. In their study, suPAR was associated with early systolic and diastolic myocardial impairment, measured via echocardiographic techniques [12]. By demonstrating that suPAR is predictive of impaired myocardial relaxation, an early manifestation diastolic dysfunction commonly associated with ageing, our current study builds on this emerging link between suPAR and diastolic function, particularly as assessed by echocardiography. Diabetes mellitus was not a significant predictor of myocardial function in our study, in contrast to Theilade et al.'s study [12] that was prespecified to study suPAR among diabetic patients. Further, we had acquired echocardiographic images simultaneously with blood sampling at the same study visit, possibly eliminating potential clinical events intervening between assessment of myocardial function and blood sampling that may inaccurately depict the relationship between suPAR and myocardial function. In contrast to Theilade's study [12] that had a median interval of 116 days between echocardiography acquisition and blood sampling, the observed value of suPAR in predicting myocardial function in our study is unlikely influenced by other unknown systemic disease processes. This is especially pertinent in studies that evaluate suPAR as a potential biomarker of disease, because suPAR may represent general states of ill-health. SuPAR predicts a broad spectrum of conditions including cancer, cardiovascular disease, diabetes, kidney disease and mortality [13], purportedly as a result of its role as an endpoint effector of the immune system.

A proinflammatory microenvironment and low-grade inflammation mediated by mechanical and humoral factors, and driven by oxidative stress is commonly described in ageing. These proinflammatory mechanisms drive age-associated arterial remodelling [4], as a result of processes that alter cellular and matrix structure and 
Table 1 Baseline clinical characteristics of study participants

\begin{tabular}{|c|c|c|c|c|c|}
\hline & & $\begin{array}{l}\text { Impaired myocardial } \\
\text { function }(n=74)\end{array}$ & $\begin{array}{l}\text { Preserved myocardial } \\
\text { function }(n=46)\end{array}$ & Total $(n=120)$ & $P$-value \\
\hline Age (years) & & $73.3 \pm 4.0$ & $66.5 \pm 13.4$ & $70.3 \pm 9.4$ & 0.0001 \\
\hline SUPAR & & $3.9 \pm 2.2$ & $3.0 \pm 1.4$ & $3.5 \pm 2.0$ & 0.015 \\
\hline Female gender & & 29 (39.2\%) & $16(34.8 \%)$ & 45 (37.5\%) & 0.63 \\
\hline Body mass index $\left(\mathrm{kg} / \mathrm{m}^{2}\right)$ & & $23.7(2.9)$ & $23.3(3.4)$ & $23.5(3.1)$ & 0.45 \\
\hline Ever smoker & & $20(27.0 \%)$ & $7(15.2 \%)$ & 27 (22.5\%) & 0.13 \\
\hline Hypertension & & $41(55.4 \%)$ & $24(52.2 \%)$ & $65(54.2 \%)$ & 0.73 \\
\hline Dyslipidemia & & $41(55.4 \%)$ & $22(47.8 \%)$ & $63(52.5 \%)$ & 0.42 \\
\hline Diabetes mellitus & & $20(27.0 \%)$ & $5(10.9 \%)$ & $25(20.8 \%)$ & 0.034 \\
\hline Systolic blood pressure & & $155.4(39.3)$ & $145.0(13.5)$ & $151.4(39.3)$ & 0.088 \\
\hline Diastolic blood pressure & & $75.9(10.3)$ & $76.0(12.2)$ & $75.9(10.3)$ & 0.96 \\
\hline \multirow[t]{2}{*}{ Pulse } & $<69$ & $25(33.8 \%)$ & $22(47.8 \%)$ & 47 (39.2\%) & 0.13 \\
\hline & $\geq 69$ & 49 (66.2\%) & $24(52.2 \%)$ & $73(60.8 \%)$ & \\
\hline Interventricular septum thickness at end diastole (IVSd) (cm) & & $0.9 \pm 0.2$ & $0.8 \pm 0.1$ & $0.8 \pm 0.2$ & 0.012 \\
\hline Interventricular septum thickness at end systole (IVSs) (cm) & & $1.3 \pm 0.3$ & $1.2 \pm 0.3$ & $1.3 \pm 0.3$ & 0.35 \\
\hline Left ventricular internal diameter end diastole (LVIDd) (cm) & & $4.5 \pm 0.5$ & $4.6 \pm 0.5$ & $4.5 \pm 0.5$ & 0.14 \\
\hline Left ventricular internal diameter end systole (LVIDs) (cm) & & $2.5 \pm 0.4$ & $2.6 \pm 0.4$ & $2.5 \pm 0.4$ & 0.027 \\
\hline Left ventricular posterior wall end diastole (LVPWd) $(\mathrm{cm})$ & & $0.8 \pm 0.1$ & $0.7 \pm 0.1$ & $0.8 \pm 0.1$ & 0.074 \\
\hline Left ventricular posterior wall end systole (LVPWs)(cm) & & $1.4 \pm 0.2$ & $1.4 \pm 0.2$ & $1.4 \pm 0.2$ & 0.62 \\
\hline Left ventricular outflow tract (LVOT) (cm) & & $2.1 \pm 0.1$ & $2.0 \pm 0.2$ & $2.1 \pm 0.1$ & 0.49 \\
\hline Aortic diameter (Ao) (cm) & & $3.2 \pm 0.4$ & $3.0 \pm 0.5$ & $3.1 \pm 0.5$ & 0.058 \\
\hline Left atrium $(\mathrm{LA})(\mathrm{cm})$ & & $3.7 \pm 0.5$ & $3.6 \pm 0.6$ & $3.7 \pm 0.6$ & 0.17 \\
\hline Left ventricular ejection fraction (LVEF) (\%) & & $75.3 \pm 6.7$ & $72.7 \pm 7.0$ & $74.3 \pm 6.9$ & 0.047 \\
\hline Left ventricular fractional shortening (LV FS) (\%) & & $44.3 \pm 6.0$ & $42.5 \pm 6.3$ & $43.6 \pm 6.2$ & 0.13 \\
\hline Left ventricular mass (LVM) (grams) & & $132.5 \pm 41.2$ & $127.6 \pm 33.1$ & $130.6 \pm 38.2$ & 0.51 \\
\hline Left ventricular mass index (LVMI) (grams/m²) & & $82.0 \pm 23.3$ & $78.5 \pm 19.2$ & $80.8 \pm 21.9$ & 0.43 \\
\hline Left atrial volume (LAV) (ml) & & $38.0 \pm 13.5$ & $36.7 \pm 13.6$ & $37.5 \pm 13.5$ & 0.62 \\
\hline Left atrial volume index (LAVI) $\left(\mathrm{ml} / \mathrm{m}^{2}\right)$ & & $23.6 \pm 7.9$ & $22.3 \pm 8.0$ & $23.1 \pm 7.9$ & 0.42 \\
\hline Peak velocity flow in early diastole E (MV E Peak) (m/s) & & $0.6 \pm 0.1$ & $0.7 \pm 0.2$ & $0.7 \pm 0.2$ & 0.0001 \\
\hline $\begin{array}{l}\text { Peak velocity flow in late diastole by atrial contraction } \\
\text { A (MV A Peak) (m/s) }\end{array}$ & & $0.8 \pm 0.2$ & $0.7 \pm 0.2$ & $0.8 \pm 0.2$ & 0.0014 \\
\hline Ratio of MV E peak/MV A peak (E/A Ratio) & & $0.8 \pm 0.2$ & $1.1 \pm 0.4$ & $0.9 \pm 0.3$ & $<0.0001$ \\
\hline Deceleration time (DT) (m/s) & & $218.0 \pm 39.0$ & $198.0 \pm 37.1$ & $210.3 \pm 39.4$ & 0.0064 \\
\hline Pulmonary artery systolic pressure (PASP) (mmHg) & & $26.9 \pm 6.1$ & $27.0 \pm 6.7$ & $27.0 \pm 6.3$ & 0.94 \\
\hline Pulmonary vein systolic velocity (PV-S) (cm/s) & & $59.2 \pm 11.7$ & $59.1 \pm 14.7$ & $59.2 \pm 11.7$ & 0.96 \\
\hline Pulmonary vein diastolic velocity (PV-D) (cm/s) & & $46.8 \pm 15.0$ & $51.1 \pm 14.1$ & $48.5 \pm 14.7$ & 0.13 \\
\hline Pulmonary vein flow at atrial contraction (PV-Ar) (ms) & & $92.8 \pm 17.6$ & $95.1 \pm 23.4$ & $93.7 \pm 20.0$ & 0.55 \\
\hline Mitral inflow duration at atrial contraction (MV A duration) (ms) & & $115.8 \pm 15.6$ & $113.8 \pm 17.2$ & $115.0 \pm 16.2$ & 0.51 \\
\hline Peak systolic septal mitral annular velocity (Septal S') (m/s) & & $0.08 \pm 0.01$ & $0.08 \pm 0.01$ & $0.08 \pm 0.01$ & 0.72 \\
\hline Peak early diastolic septal mitral annular velocity (Septal E') (m/s) & & $0.06 \pm 0.02$ & $0.09 \pm 0.02$ & $0.07 \pm 0.02$ & $<0.0001$ \\
\hline Septal mitral annular velocity during atrial contraction (Septal A') $(\mathrm{m} / \mathrm{s})$ & & $0.1 \pm 0.02$ & $0.1 \pm 0.02$ & $0.1 \pm 0.02$ & 0.69 \\
\hline Ratio of MV E peak/Septal E' & & $10.1 \pm 2.7$ & $9.4 \pm 4.4$ & $9.8 \pm 3.5$ & 0.28 \\
\hline
\end{tabular}

Continuous data are shown as mean \pm SD 
Table 2 Univariable and multivariable association with impaired myocardial function

\begin{tabular}{|c|c|c|c|c|c|c|c|}
\hline & & Univariate & & Multivariate & & & \\
\hline & & Unadjusted OR (95\% Cl) & $P$-value & Adjusted OR (95\% Cl) & $P$-value & Adjusted OR of suPAR $(95 \% \mathrm{Cl})$ & $P$-value \\
\hline SUPAR & $<3.0$ & 1 & 0.002 & - & & - & \\
\hline & $\geq 3.0$ & $3.4(1.6,7.4)$ & & & & & \\
\hline Age (years) & $\leq 70$ & 1 & 0.003 & 1 & & & \\
\hline & $>70$ & $3.6(1.6,8.5)$ & & $2.8(1.1,6.7)$ & 0.026 & $2.7(1.2,6.1)$ & 0.016 \\
\hline Gender & Male & 1 & 0.63 & - & & - & \\
\hline & Female & $1.2(0.6,2.6)$ & & & & & \\
\hline Body mass index $\left(\mathrm{kg} / \mathrm{m}^{2}\right)$ & & $1.05(0.9,1.2)$ & 0.44 & - & & - & \\
\hline Ever smoker & & $2.1(0.8,5.4)$ & 0.14 & - & & - & \\
\hline Hypertension & & $1.1(0.5,2.4)$ & 0.73 & - & & - & \\
\hline Dyslipidemia & & $1.4(0.6,2.8)$ & 0.42 & - & & - & \\
\hline Diabetes mellitus & & $3.04(1.1,8.8)$ & 0.040 & $2.4(0.8,7.1)$ & 0.12 & $3.0(1.4,6.7)$ & 0.006 \\
\hline Systolic blood pressure & & $1.03(1.001,1.1)$ & 0.041 & $1.02(1.0,1.05)$ & 0.14 & $3.0(1.3,6.5)$ & 0.007 \\
\hline Diastolic blood pressure & & $1.0(1.0,1.03)$ & 0.96 & - & & - & \\
\hline Pulse & $<69$ & 1 & 0.13 & - & & - & \\
\hline & $\geq 69$ & $1.8(0.8,3.8)$ & & & & & \\
\hline
\end{tabular}

function. Prolonged contraction of the left ventricle against a stiff aorta results in incomplete myocardial relaxation at the onset of diastole, reducing early left ventricular filling rate. Other structural changes occurring within left ventricular tissue with ageing may also contribute to this reduction in peak LV filling rate [5]. It is possible that the process of inflammation in ageing is mechanistically linked to suPAR release. Proinflammatory cytokines such as tumor necrosis factor- $\alpha$ that induce left ventricular dysfunction may simultaneously stimulate release of suPAR from activated neutrophils, monocytes and endothelial cells via proteolytic cleavage from the cell surface into the plasma [4]. This inflammatory process is maintained by suPAR because it acts as a

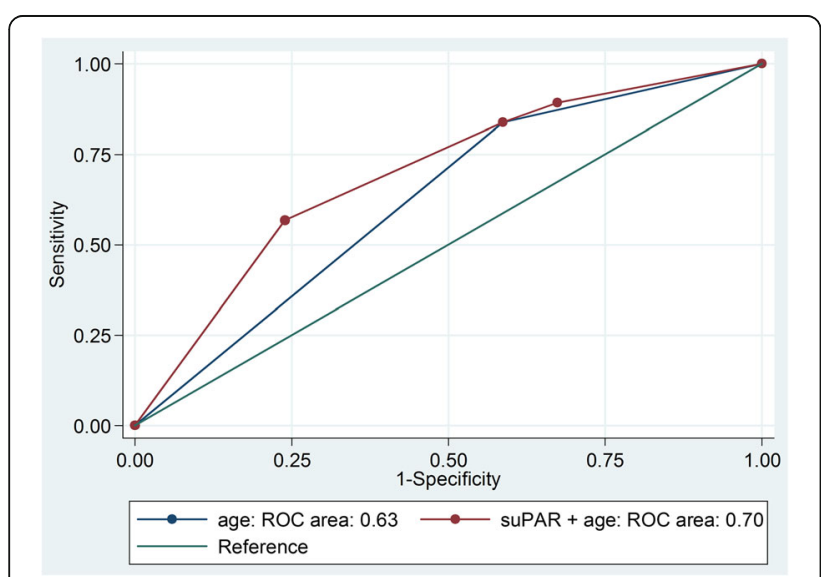

Fig. 1 Receiver operating characteristic (ROC) curves for discriminating between preserved myocardial function and impaired myocardial function using age (blue line) or age plus suPAR (red line) chemotactic agent that promotes recruitment of immune cells to sites of acute inflammation [10]. The associations between suPAR and subclinical myocardial function in our study provides hypothesis-generating data to suggest a common inflammatory basis for the observed associations [14].

The question as to whether suPAR is a surrogate marker or a contributor to impaired myocardial function associated with the ageing heart requires future work. We believe our study is the first to study suPAR in relation to the ageing heart, particularly in exploring the added ability of suPAR over age in predicting heart ageing.

SuPAR has recently been shown to be associated with hard outcome events in CVD cohorts. A recent study of chronic heart failure patients demonstrated prognostic value of suPAR in predicting all-cause mortality [8]. Another population-based study of elderly participants associated elevated levels of suPAR with increased incidence of incident cardiovascular events [7]. Our results suggest that suPAR may actually be used upstream prior to clinical heart disease, for identifying the ageing heart. Further, future therapeutic efforts that target anti-inflammatory processes, may use suPAR to identify patients for earlier or intensified anti-inflammatory therapies. Treatment effects could also theoretically be monitored through alterations in suPAR levels during treatment [9].

Finally, the relative stability of suPAR in plasma better reflects the state of chronic immune activation compared to other inflammatory biomarkers, such as Creactive protein (CRP). SUPAR is less inducible than CRP with no major variation in blood and circadian levels [13]. This is particularly relevant for community 
based studies, such as ageing studies, where blood draws are taken at random times of the day and blood is usually stored prior to analysis necessitating frequent thawing and freezing cycles $[6,13]$.

The current study has limitations. The analyses are cross-sectional and hence we are unable to determine causality. The sample size is relatively small and participation is voluntary, introducing possible biases in participant selection and inclusion of participants who were generally more interested in their health status (i.e, healthier). Even so, the true relationship between suPAR and myocardial function may only be underestimated, and not overestimated. uPAR has three domains D1, D2 and D3. Soluble forms are composed of D1-D2-D3 and D2-D3 which arise from cleavage of GPI-anchor. Further cleavage of the linker region gives rise to D1 domain [15]. The assay used in our analysis could not differentiate between the forms of suPAR. Hence, we are unable to examine associations of different subtypes of suPAR to myocardial function.

\section{Conclusions}

In this community-based study of participants without cardiovascular disease, we demonstrate additional ability of suPAR, over age, to predict impaired myocardial relaxation. Future studies investigating whether suPAR has a direct role in the pathogenesis of diastolic dysfunction or is merely a bystander reflecting chronic inflammation are warranted.

\begin{abstract}
Abbreviations
Ao: Aortic diameter; CV: Cardiovascular; DT: Deceleration time; IVSd: Interventricular septum thickness at end diastole; IVSs: Interventricular septum thickness at end systole; LA: Left atrium; LAV: Left atrial volume; LAVI: Left atrial volume index; LV FS: Left ventricular fractional shortening; LVEF: Left ventricular ejection fraction; LVIDd: Left ventricular internal diameter end diastole; LVIDs: Left ventricular internal diameter end systole; LVM: Left ventricular mass; LVMI: Left ventricular mass index; LVOT: Left ventricular outflow tract; LVPWd: Left ventricular posterior wall end diastole; LVPWs: Left ventricular posterior wall end systole; MV A duration: Mitral inflow duration at atrial contraction; MV A Peak: Peak velocity flow in late diastole by atrial contraction A; MV E Peak: Peak velocity flow in early diastole E; PASP: Pulmonary artery systolic pressure; PV-Ar: Pulmonary vein flow at atrial contraction; PV-D: Pulmonary vein diastolic velocity; PVS: Pulmonary vein systolic velocity; Septal A': Septal mitral annular velocity during atrial contraction; Septal E': Peak early diastolic septal mitral annular velocity; Septal S': Peak systolic septal mitral annular velocity; sUPAR: soluble urokinase plasminogen activator receptor
\end{abstract}

\section{Acknowledgements}

Not applicable.

\section{Consent for publication}

Not applicable.

\section{Funding}

The Cardiac Aging Study has received funding support from the National Medical Research Council of Singapore (NMRC/TA/0031/2015), Hong Leong Foundation and Edwards Lifesciences. Participants from the Singapore Chinese Health Study were supported by the United States National Institutes of Health (NIH R01 CA144034 and UM1 CA182876). C.Cheung was supported by a Nanyang Assistant Professorship, Singapore, and an IMCB Independent Fellowship (A*STAR). The funder had no role in the design and conduct of the study; collection; management, analysis, and interpretation of the data; and preparation, review, or approval of the manuscript.

\section{Availability of data and materials}

The datasets used and analyzed during the present study are available from the corresponding author on request.

\section{Authors' contributions}

AK, BV, CC: designed the research; FG, AK, analysed data. AK, RST, JIW, SJMC acquired the data and interpreted the data and gave final approval of the version to be published; AK, BV, FG, RST, LLYT, BMHK, JMY, WPK, CC drafted the manuscript, revised the manuscript critically and gave final approval of the version to be published.

\section{Ethics approval and consent to participate}

Written informed consent was obtained from participants upon enrolment The SingHealth Centralised Institutional Review Board had approved the study protocol (2014/628/C)

\section{Competing interests}

The authors declare that they have no competing interests.

\section{Publisher's Note}

Springer Nature remains neutral with regard to jurisdictional claims in published maps and institutional affiliations.

\section{Author details}

${ }^{1}$ National Heart Centre Singapore, 5 Hospital Drive, Singapore 169609, Singapore. ${ }^{2}$ Duke-NUS Medical School, Singapore, Singapore. ${ }^{3}$ Institute of Molecular and Cell Biology, Agency for Science, Technology and Research (A*STAR), Singapore, Singapore. ${ }^{4}$ Division of Cancer Control and Population Sciences, University of Pittsburgh Cancer Institute, Pittsburgh, PA, USA. ${ }^{5}$ Department of Epidemiology, Graduate School of Public Health, University of Pittsburgh, Pittsburgh, PA, USA. ${ }^{6}$ Saw Swee Hock School of Public Health, National University of Singapore, Singapore, Singapore. ${ }^{7}$ Lee Kong Chian School of Medicine, Nanyang Technological University, Singapore, Singapore.

Received: 23 August 2017 Accepted: 15 November 2017

Published online: 28 November 2017

\section{References}

1. Lakatta EG, Levy D. Arterial and cardiac aging: major shareholders in cardiovascular disease enterprises: part l: aging arteries: a "set up" for vascular disease. Circulation. 2003;107(1):139-46.

2. Lakatta EG. Arterial and cardiac aging: major shareholders in cardiovascular disease enterprises: part III: cellular and molecular clues to heart and arterial aging. Circulation. 2003;107(3):490-7.

3. Lakatta EG, Levy D. Arterial and cardiac aging: major shareholders in cardiovascular disease enterprises: part II: the aging heart in health: links to heart disease. Circulation. 2003:107(2):346-54.

4. Mann DL. Innate immunity and the failing heart: the cytokine hypothesis revisited. Circ Res. 2015;116(7):1254-68.

5. Lakatta EG. Cardiovascular regulatory mechanisms in advanced age. Physiol Rev. 1993;73(2):413-67

6. Riisbro R, Christensen IJ, Hogdall C, Brunner N, Hogdall E. Soluble urokinase plasminogen activator receptor measurements: influence of sample handling. Int J Biol Markers. 2001:16(4):233-9.

7. Persson M, Engstrom G, Bjorkbacka H, Hedblad B. Soluble urokinase plasminogen activator receptor in plasma is associated with incidence of CVD. Results from the Malmo diet and cancer study. Atherosclerosis. 2012 220(2):502-5.

8. Koller L, Stojkovic S, Richter B, Sulzgruber P, Potolidis C, Liebhart F, et al. Soluble Urokinase-type plasminogen activator receptor improves risk prediction in patients with chronic heart failure. JACC Heart Fail. 2017:5(4):268-77.

9. Lyngbaek S, Marott JL, Moller DV, Christiansen M, Iversen KK, Clemmensen PM, et al. Usefulness of soluble urokinase plasminogen activator receptor to predict repeat myocardial infarction and mortality in patients with STsegment elevation myocardial infarction undergoing primary percutaneous intervention. Am J Cardiol. 2012;110(12):1756-63. 
10. Pliyev BK. Activated human neutrophils rapidly release the chemotactically active D2D3 form of the urokinase-type plasminogen activator receptor (UPAR/CD87). Mol Cell Biochem. 2009;321(1-2):111-22.

11. Hankin JH, Stram DO, Arakawa K, Park S, Low SH, Lee HP, et al. Singapore Chinese health study: development, validation, and calibration of the quantitative food frequency questionnaire. Nutr Cancer. 2001;39(2):187-95.

12. Theilade S, Rossing P, Eugen-Olsen J, Jensen JS, Jensen MT. suPAR level is associated with myocardial impairment assessed with advanced echocardiography in patients with type 1 diabetes with normal ejection fraction and without known heart disease or end-stage renal disease. Eur Endocrinol. 2016 Jun;174(6):745-53.

13. Eugen-Olsen J, Andersen O, Linneberg A, Ladelund S, Hansen TW, Langkilde A, et al. Circulating soluble urokinase plasminogen activator receptor predicts cancer, cardiovascular disease, diabetes and mortality in the general population. J Intern Med. 2010;268(3):296-308.

14. Parish RC, Evans JD. Inflammation in chronic heart failure. Ann Pharmacother. 2008:42(7):1002-16.

15. Blasi F, Carmeliet P. UPAR: a versatile signalling orchestrator. Nat Rev Mol Cell Biol. 2002;3(12):932-43.

\section{Submit your next manuscript to BioMed Central} and we will help you at every step:

- We accept pre-submission inquiries

- Our selector tool helps you to find the most relevant journal

- We provide round the clock customer support

- Convenient online submission

- Thorough peer review

- Inclusion in PubMed and all major indexing services

- Maximum visibility for your research

Submit your manuscript at www.biomedcentral.com/submit 